\title{
Automation using Plc and IIOT Monitoring in Jaggery Preparation
}

\author{
G.Jayanthi, S.Aaradhika, S.Swathy, N.Mathumitha
}

\begin{abstract}
Jaggery is a natural sugar which a sweetening agent and is also called as gur or panela. It is first made into a semisolid paste by evaporating the sugarcane juice. The aim of this paper is to develop an automated process for Jaggery preparation using PLC and monitored by IIOT (Industrial Internet Of Things). The process consists of pushbuttons, Temperature sensor (LM35), Level sensor (Ultrasonic level transmitter), IR sensor as input devices which are interfaced with the Siemens PLC(CPU314-2C). The PLC controls the following output devices such as crusher motor, conveyor motor, scum motor and valves (pneumatic actuator control valve).The process parameters are uploaded into the cloud for monitoring using IIOT. The trend graphs are generated using MindSphere software (MindConnect Nano). The system also provides fault detection through alert messages and e-mails. The protocol used for IIOT is Transmission Control Protocol (TCP). All the five stages such as crushing, filtering, heating, cooling and molding of the Jaggery preparation process are automated. Thus, a hygienic and fully automatic PLC based Jaggery production system with fault detection capability is developed through this project.
\end{abstract}

KEYWORDS: PLC, IIOT, MindSphere, MindConnect Nano, Trend plot, Fault detection, TCP.

\section{INTRODUCTION}

\subsection{General}

Jaggery is a natural sweetener obtained from the concentrated sugarcane juice. It has health benefits since it is loaded with antioxidants and minerals like zinc and selenium.

Jaggery is used in making of sweets and savory dishes in cuisines of India, Sri Lanka, Nepal, Bangladesh, Afghanistan and Iran. In South India, it is used to balance pungency of spicy food. In Tamil Nadu, Jaggery is well known as sweetener. For example, in preparation of Chakkarai Pongal dish during Pongal festival. Jaggery has religious significance to Hindus all over India. Many of the festivals involve the offering of Jaggery to deities during worship.

Benefits of Sugarcane:

- Detoxifies the body

- Prevents anemia due to rich iron contents

- Boosts Immunity

- Blood purifier

- Treat flu-like symptoms

- Relieves joint pain

Revised Manuscript Received on November 08, 2019.

* Correspondence Author

G.Jayanthi, Department Of Instrumentation and Control Engineering, Sri Sairam Engineering College,Chennai,Tamil Nadu,India.

S.Aaradhika, Department Of Instrumentation and Control Engineering, Sri Sairam Engineering College,Chennai,Tamil Nadu,India.

S.Swathy, Department Of Instrumentation and Control Engineering, Sri Sairam Engineering College,Chennai,Tamil Nadu,India.

N.Mathumitha, Department Of Instrumentation and Control Engineering, Sri Sairam Engineering College,Chennai,Tamil Nadu,India.

\subsection{Programmable Logic Controller}

Programmable Logic Controller (PLC) is a digital computer which can be adapted for controlling manufacturing process. PLC is one of the most common tools used for automation purpose. PLC uses a programmable memory to store instructions and execute specific functions that include ON/OFF control, counting, timing, sequence and data handling. It was designed to replace relay logic system. PLC's are defined and governed by the IEC 61131-3 standard (International Electro technical Commission). PLC is known to tolerate severe conditions such as dust, moisture, heat,cold etc. the design of most PLC's is similar to that of other computers. For example, simple process operation uses Ladder Logic. Instruction List commanding is used as it provides better execution of program speed as they are basic assembly level language. Different programming languages used are Sequential Flow Chart, Functional Block Diagram and Structured Text. Programming language may vary depending upon the area of operation. Some features of PLC are communication capability, flexibility, increases reliability, easier to troubleshoot and faster response. Various brands used in PLC are Siemens, Keyence, Omron, Danfoss, Allen Bradley, Delta etc.

\subsection{Industrial Internet Of Things}

IIOT (Industrial Internet of Things or Industry 4.0) is use of IOT (Internet of Things) to enhance manufacturing industry or industrial process. It transforms the manufacturing by enabling the acquisition and accessibility of greater amount of data at greater speed and more efficient. IIOT is implemented by leveraging intelligent, connected devices in companies. The emerging standard for IIOT is MQTT (Message Queuing Telemetry Transfer) protocol due to its publish/subscribe model, lightweight overhead and bidirectional capabilities with their unlimited data acquisition. MQTT is designed for constrained devices with low bandwidth, hence it is a perfect solution for IOT applications. It establishes communication between multiple devices by allowing to send commands to control outputs, read and publish the data from the devices.

The basic concepts of MQTT are:

- Publish/Subscribe

- Message

- Topics

- Broker

Benefits of IIOT:

It helped to greatly improve

- Connectivity

- Efficiency

- Scalability

- Time saving 
- Cost saving due to their predictive maintenance, improved safety

- To get a full accurate view which helps to make better decision

\section{LITERATURE REVIEW}

Jaggery (Gur or Panela) is a natural sweetener, made by evaporating water from cane juice, thereby making it highly concentrated as a thick semisolid paste. Jaggery being the purest form as it is free from chemicals is known as a substitute for sugar which is manufactured chemically. Conventionally, Jaggery manufacturing is a tedious and unhygienic process, involving risky working conditions. This paper elaborates a whole new process for Jaggery manufacturing, providing a hygienic and fully automated manufacturing environment, hence reducing the manpower involved. The main controlling and consoling unit is a PLC (Programmable Logic Controller). PLC interfaces all the inputs and the outputs. The Switches and the Sensors are the inputs while the Valves, Machines, LED's, Conveyers, etc. are the Outputs. Jaggery production essentially involves following steps viz. harvesting of raw Sugarcane, Pre-cleaning, Crushing, Filtration, Boiling, Concentration, evaporation and finally filling them into moulds. All the machines are controlled by the PLC and proper working of the whole plant is monitored through SCADA (Supervisory Control and Data Acquisition). Various additives are added for enhancing the quality and clarification of Sugarcane juice at the time of boiling. Proper automated filling in moulds is done to give it a proper shape. The process for Jaggery manufacturing will therefore result in both, enhancing the rate of production and the quality of Jaggery and thus Jaggery production will be more profitable for the Sugarcane growers. Thus, by using automated technologies, many commercial availabilities become needs of the hour to sustain future profitability in Jaggery production, thereby having a direct effect on health and wealth of people.

\section{PROPOSED ALGORITHM}

This paper proposes that when cleaned tons of sugarcanes are sensed by the Infra-Red Sensor, conveyor motor starts and canes are rolled over and it is crushed by the crusher motor to extract the sugarcane juice. These juices are filtered in the storage tank to remove the scum. When the level of the storage tank is sensed about $70 \%-80 \%$, it is pumped to the evaporator using a single phase pump. Here, the steam is let into the evaporator to heat the cane juice and the temperature is maintained and monitored using the temperature sensor (LM35 or PT100) appropriately. The heated solution is then led to the condenser through the control valve and it is cooled to an appropriate temperature and it is formed into a semisolid paste. This paste is led through the valve into the moulds running over the conveyor which consists of sensor to moulds. The temperature parameter and level parameter are uploaded into the cloud for monitoring. When fault occurs, it is alerted through e-mail. The monitored output is shown as trend graphs.

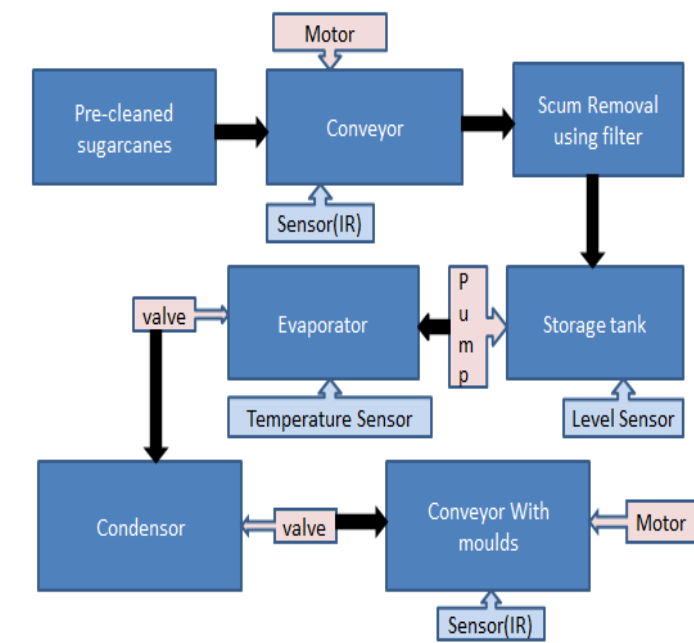

Figure 3.1 General block diagram

\subsection{Process Explanation}

$>$ Pre-Requisites

The condenser fans are turned on and the following values are to be entered in the PID block before the commencement of process :

$\checkmark$ P_SLT:ON(1)

$\checkmark$ PV_IN: Present temperature

$\checkmark$ SP_INT: Required temperature $\left(125^{\circ} \mathrm{C}\right)$

$\checkmark$ GAIN: 1

$\checkmark$ LNM_HLM: $150^{\circ} \mathrm{C}$

$\checkmark$ LNM_LLM:-50 ${ }^{\circ} \mathrm{C}$

$\checkmark$ PV_FAC:1

$\checkmark$ LNM_FAC:1

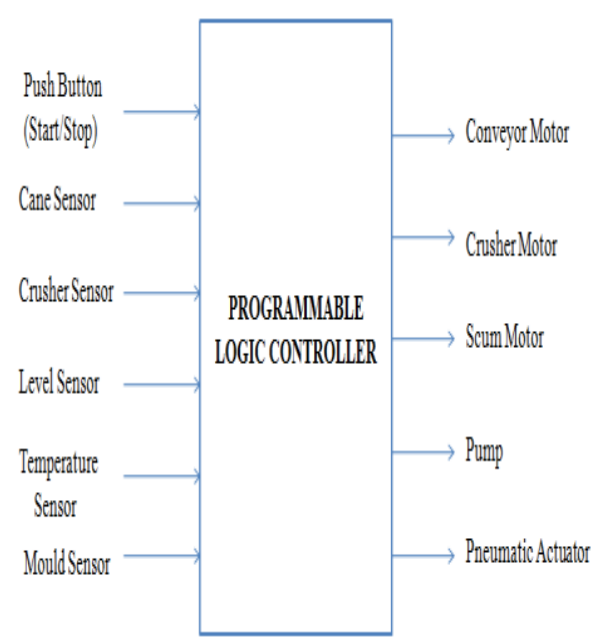

Figure 3.2 PLC I/O Block Diagram

\section{$>$ Commencement}

The process begins only when the storage tank level is less than $5 \%$. Then, the Start push button is switched on to initialize the process. When the IR sensor senses the incoming sugarcane, conveyor motor starts. When the crusher sensor is sensed, crusher motor and scum motor is simultaneously turned on till the storage tank level is less than $70 \%$.The above process is simulated using SET/RESET function to self-hold certain instruction. The process is reset only when the reset condition is 
true which helps to reduce the logic synthesis.

$>$ Storage

The crushed and filtered juice is stored in storage tank. The sugarcane pump starts pumping only when level is between $5 \%-70 \%$. The juice is pumped to the evaporator.

$>$ Heating

For the purpose of heating the juice, evaporator is to used . It is heated to $125^{\circ} \mathrm{C}$ using steam and the steam inlet valve is controlled by the PID. When the juice is heated to appropriate temperature it is led to the condenser through the valve.

$>$ Cooling

The concentrated juice is cooled using the fans in the condenser to the required temperature.

$>$ Molding

When mould sensor senses the moulds, mould valve opens for 5 second to fill the cooled paste using timer instruction.

\section{SOFTWARE DESCRIPTION}

\subsection{Mindsphere}

MindSphere is the Siemens Industrial IoT operation system comprising the core cloud services and applications (Core Apps, Mind Apps), whereas the Mind Connect Element provides secure and easy connectivity from the field or machine to Mind Sphere. In MindSphere, submitted data by a MindConnect Element is processed and further stored for analysis and management purposes. MindSphere provides the means to monitor asset status as well as support maintenance and services. This method requires sensor data, the tools to collect and transfer data as well as intelligent software that offers the monitoring and support functions.

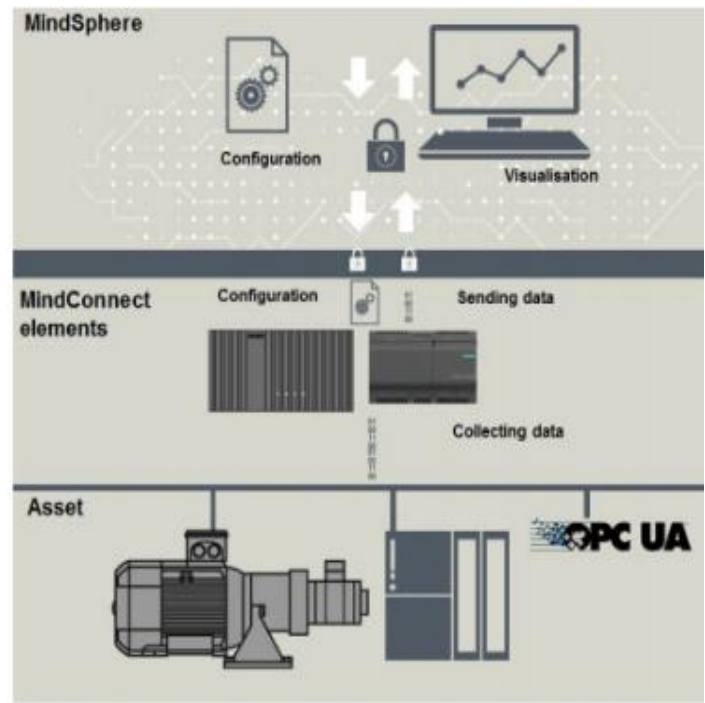

Figure 4.1 Overview of Mindsphere

\section{Requirements of Mindsphere}

- Internet Connection and browser are for online User Interface. Browser supporting HTML5 (recommended are Firefox 43.0, Google Chrome 47.0, Safari iPad)

- Internet Connection for MindConnect Nano/IoT2040 device. Provides standard HTTPS capabilities for MindConnect Nano/IoT2040 - outbound HTTPs connections on port 443.

- Link to the online Launchpad along with user and password data as it was submitted by Siemens.
- Devices (PC, tablet etc.) with minimum screen resolution of $1024 \times 768$

\subsection{Step 7}

STEP 7 is a standard software package used for configuring and programming SIMATIC programmable logic controllers(PLC). It is a part of the SIMATIC industry software. The wide ranges of functions offered by STEP7 are:

- An opportunity to assign parameters to function modules and communication processors.

- Forcing and multi-computing mode.

- Global data communication.

- Event-driven data transfer using communication function blocks.

- Configuring connections.

The applications of STEP7 include:

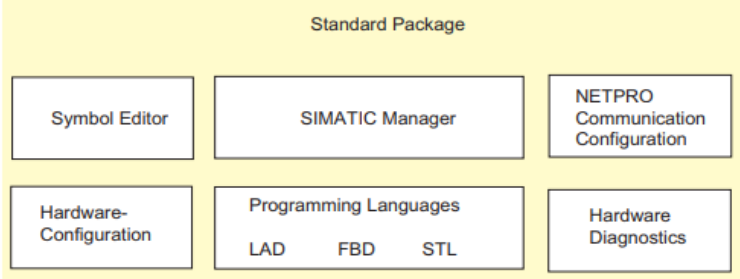

Figure 4.2 Application of Step 7

\section{EXPERIMENT AND RESULT}

\subsection{Continous Temperature control using PID}

The below figure 5.1 represents the opening/closing of valve depending on the temperature of the evaporator.

PID CALCULATION

PID output $=$ Offset $+K_{p}\left(e_{p}\right)$

Where $\mathrm{K}_{\mathrm{p}}$ is Gain , $\mathrm{e}_{\mathrm{p}}$ is Proportional Error

Example:

When Offset $=0 ; \mathrm{K}_{\mathrm{p}}=1 ; \mathrm{e}_{\mathrm{p}}=75$

Then, PID Output $=0+1(75)=75 \%$

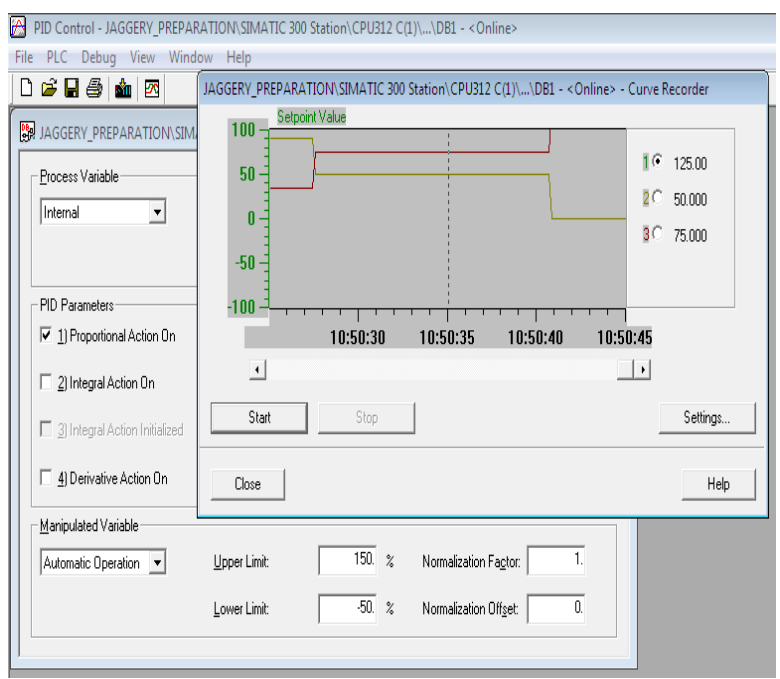

Figure 5.1 PID Graph 
This $75 \%$ indicates the percentage of valve opening to provide the required steam for the evaporator.As the PID output changes,percentage of valve opening also changes.

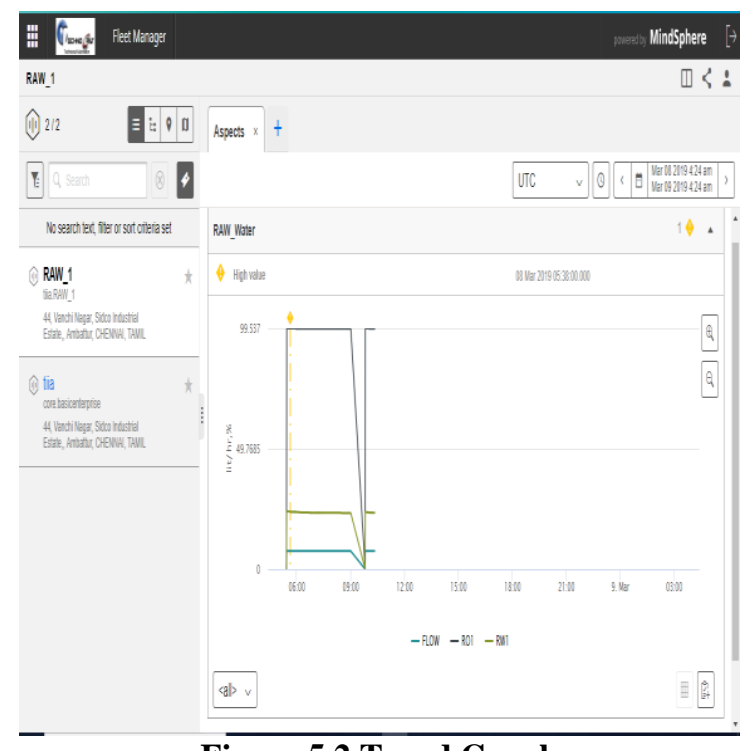

Figure 5.2 Trend Graph

\section{CONCLUSION}

Thus a hygienic fully automated PLC based jaggery production system with fault detection capability was developed through this project.The MindSphere acts as the web server to store and analyse the process parameters. The Mindsphere provides master login to the higher authorities and user login for operating persons.It provides end to end encryption for data security.The daily, monthly, quarterly reports are generated to monitor the continous analysis of the process parameters.If any fault occurs, it is intimated through alert mails.

\section{REFERENCES}

1. Abhishek Jain, Rohit Parakh Jain and Sanjana Lamba (2017)"APPLICATION OF PLC AND SCADA IN HIGH QUALITY MEDIUM SCALE JAGGERY PRODUCTION PROCESS" International Journal of Latest Trends in Engineering and Technology (IJLET)

2. Prof. Niranjan M Madhukar N Ashwini A Muddsar J Saish M (2017) "IOT Based Industrial Automation" - IOSR Journal of Computer Engineering (IOSR-JCE) National Conference

3. Prof. Yogesh Shankar kumbhar (2016) "Design \& Manufacturing of Automation system for Jaggery Plant" - International Research Journal of Engineering and Technology (IRJET)

4. F.D. Petruzella, Programmable Logic Controllers, Tata Mc-Graw Hill, Third edition, 2010.

1. Hwaiyu Geng, "THE INDUSTRIAL INTERNET OF THINGS (IIoT)," in Internet of Things and Data Analytics Handbook , , Wiley, 2017, pp.41-81 